\title{
Hamilton cycles in planar locally finite graphs
}

\author{
Henning Bruhn Xingxing $\mathrm{Yu}$
}

\begin{abstract}
A classical theorem by Tutte assures the existence of a Hamilton cycle in every finite 4-connected planar graph. Extensions of this result to infinite graphs require a suitable concept of an infinite cycle. Such a concept was provided by Diestel and Kühn, who defined circles to be homeomorphic images of the unit circle in the Freudenthal compactification of the (locally finite) graph. With this definition we prove a partial extension of Tutte's result to locally finite graphs.
\end{abstract}

\section{Introduction}

While Hamilton cycles have been investigated intensively in finite graphs, comparatively little attention has been paid to Hamilton cycles in infinite graphs. One reason for this is that it is not entirely clear what the infinite analogon of a cycle should be.

Adomaitis [1] avoided this question by defining a graph to be hamiltonian if for every finite subset of the vertex set there is a spanning cycle. In contrast, Nash-Williams [8] addressed the problem and proposed spanning double rays as infinite analogons of Hamilton cycles. He noticed that for a spanning double ray to exist the graph needs to be 3 -indivisible. (A graph is $k$-indivisible if the deletion of finitely many vertices leaves at most $k-1$ infinite components.) Generalising the following classical result by Tutte, Nash-Williams conjectured that a 3-indivisible 4-connected planar graph contains a spanning double ray.

Theorem 1 (Tutte [11]). Every finite 4-connected planar graph has a Hamilton cycle.

Recently, Yu $[13,14,15,16,12]$ announced a proof of Nash-Williams' conjecture.

The restriction to 3-indivisible graphs is a quite serious one that at first appears unavoidable. Yet, while double rays are the obvious first choice for an infinite analogon of cycles there is a more subtle alternative, which was introduced by Diestel and Kühn $[5,6]$. They call the homeomorphic image $C$ of the unit circle in the Freudenthal compactification of a locally finite graph $G$ a circle; the subgraph $C \cap G$ is then a cycle-for finite graphs this definition coincides with the usual one. In a series of papers it has been shown that this notion is very successful and more suitable than double rays; see Diestel [3] for an introduction and a survey.

These circles overcome the restriction to 3 -indivisible graphs. Indeed, in Figure 1 we see an infinite Hamilton cycle in a graph that is not $k$-indivisible for any $k$. The example is due to Diestel and Kühn [5]. Bruhn (see [3]) conjectured that, in this sense, Theorem 1 extends to locally finite graphs: 


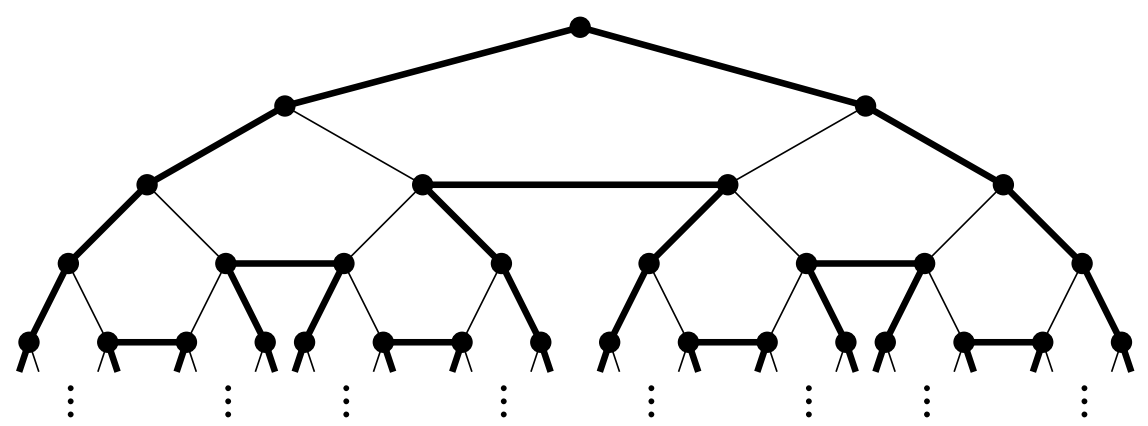

Figure 1: A Hamilton circle (drawn bold)

Conjecture 2. Let $G$ be a locally finite 4-connected planar graph. Then $G$ has a Hamilton cycle.

In this paper we will present a partial result in this direction:

Theorem 3. Let $G$ be a locally finite 6-connected planar graph that is $k$ indivisible for some finite $k \in \mathbb{N}$. Then $G$ has a Hamilton cycle.

Since submission of this paper there has been some further work on infinite Hamilton cycles. Cui, Wang and $\mathrm{Yu}[2]$ proved that a locally finite 4-connected planar graph has a Hamilton cycle, provided it has a VAP-free drawing, that is a drawing without any vertex accumulation point. This is a substantial special case of Conjecture 2. Furthermore, Georgakopoulos [7] extended Fleischner's theorem on Hamilton cycles in squares of graphs to locally finite graphs.

\section{Definitions}

In general, our notation will be that of Diestel [4]. If not otherwise noted, all graphs will be simple. A 1-way infinite path is called a ray, a 2-way infinite path is a double ray, and the subrays of a ray or double ray are its tails. Let $G=(V, E)$ be any locally finite graph. Two rays in $G$ are equivalent if no finite set of vertices separates them; the corresponding equivalence classes of rays are the ends of $G$. In several places we will implicitly use that every connected locally finite but infinite graph contains a ray, and thus an end.

We say that a finite vertex set $S$ separates an end $\omega$ from a vertex set $U$ if every ray $R \in \omega$ that starts in a vertex of $U$ meets $S$. In a similar manner, $S$ separates two ends $\omega$ and $\omega^{\prime}$ if every double ray with one tail in $\omega$ and the other in $\omega^{\prime}$ goes through $S$.

We define a topology on $G$ together with its ends, i.e. our topological space consists of all vertices, all inner points of edges and all ends of $G$. On $G$ the topology will be that of a 1-complex. Thus, the basic open neighbourhoods of an inner point on an edge are the open intervals on the edge containing that point, while the basic open neighbourhoods of a vertex $x$ are the unions of halfopen intervals containing $x$, one from every edge at $x$. For every end $\omega$ and any finite set $S \subseteq V$ there is exactly one component $C=C(S, \omega)$ of $G-S$ which contains a tail of every ray in $\omega$. We say that $\omega$ belongs to $C$, and write $\bar{C}(S, \omega)$ 
for the component $C$ together with all the ends belonging to it. Then the basic open neighbourhoods of an end $\omega$ are all sets of the form

$$
\hat{C}(S, \omega):=\bar{C}(S, \omega) \cup \stackrel{\circ}{E}(S, \omega)
$$

$\stackrel{\circ}{E}(S, \omega)$ is the set of all inner points of edges between $S$ and $C(S, \omega)$. This topological space will be denoted by $|G|$, and is also known as the Freudenthal compactification of $G$.

We will freely view $G$ either as an abstract graph or as a subspace of $|G|$, i.e. the union of all vertices and edges of $G$ with the usual topology of a 1-complex. It is not difficult to see that if $G$ is connected and locally finite, then $|G|$ is compact. Note that in $|G|$ every ray converges to the end of which it is an element.

A set $C \subseteq|G|$ is a circle if it is homeomorphic to the unit circle. Then $C$ includes every edge of which it contains an inner point, and the graph consisting of these edges and their endvertices is the cycle defined by $C$. Conversely, $C \cap G$ is dense in $C$, so every circle is the closure in $|G|$ of its cycle and hence defined uniquely by it. Note that every finite cycle in $G$ is also a cycle in this sense, but there can also be infinite cycles; see Diestel $[3,4]$ for examples and for more information. A Hamilton cycle is a cycle that contains every vertex of $G$; the corresponding circle will then cover all ends.

$G$ is called planar if there is an embedding of $G$ (as a 1-complex) in the sphere $S^{2}$. Since we work in $|G|$ rather than in $G$, the following fact is quite convenient:

Theorem 4 (Richter and Thomassen [9]). Let $G$ be a locally finite 2-connected planar graph. Then $|G|$ embeds in the sphere.

It should be noted that for an embedding $\varphi:|G| \rightarrow S^{2}$, the topological space $|G|$ coincides with the closure of $\varphi(G)$ in the sphere. We call every connected component of $S^{2} \backslash \varphi(|G|)$ a face. Its boundary is a face boundary. It is not difficult to see that each face boundary is (or, more precisely, corresponds to) the closure of a subgraph of $G$. The following lemma follows immediately from Proposition 3 in [9]:

Lemma 5. Let $G$ be a locally finite 2-connected graph with an embedding $\varphi$ : $|G| \rightarrow S^{2}$. Then the face boundaries of $\varphi(|G|)$ are circles of $|G|$.

For a subgraph $H$ of $G$, an $H$-bridge is either a chord $e \notin E(H)$ together with its endvertices both of which lie in $V(H)$ or a component $K$ of $G-H$ together with all edges between $K$ and $H$, denoted by $E(K, H)$, and their incident vertices. We say that an $H$-bridge $B$ is chordal if $E(B)$ consists of a single edge. All the vertices of an $H$-bridge $B$ in $H$ are attachments of $B$. For a subgraph $F$ of $G$, we call a path (resp. cycle) $P$ an $F$-Tutte path (resp. cycle) in $G$ if every $P$-bridge of $G$ has at most three attachments, and if every $P$-bridge containing an edge of $F$ has at most two attachments.

\section{Discussion}

The Herschel graph (see Figure 2) is a well-known example for a 3-connected planar graph without a Hamilton cycle, which shows that in a sense Theorem 1 


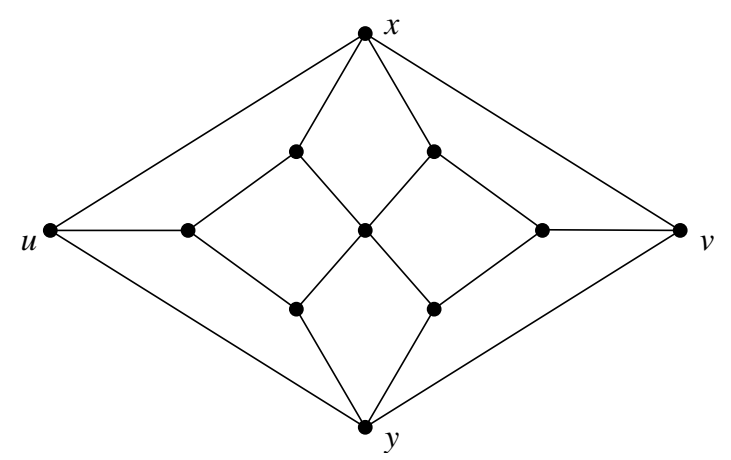

Figure 2: The Herschel graph

is best possible. In this section, let us briefly demonstrate that Conjecture 2 is also false for infinite graphs if we only assume 3-connectivity.

Consider Figure 3. There we have arranged copies of the Herschel graph (greyed) in a hexagonal grid. The copies are glued together in such a way that each copy of the vertex $v$ in Figure 2 does not receive an extra edge and thus still has degree 3 . Now assume that the resulting graph has a Hamilton cycle $C$, and consider a copy of the Herschel graph $H$ in that graph. If the Hamilton cycle enters $H$ in $u$ and leaves $H$ in either $x$ or $y$ then $C$ induces a Hamilton cycle of $H$, which is impossible. Thus, $C$ enters $H$ in $x$ and leaves it in $y$, which implies that $H$ has a Hamilton path between $x$ and $y$. However, this is impossible since both $x$ and $y$ belong to the smaller partition class in the odd bipartite graph $H$.

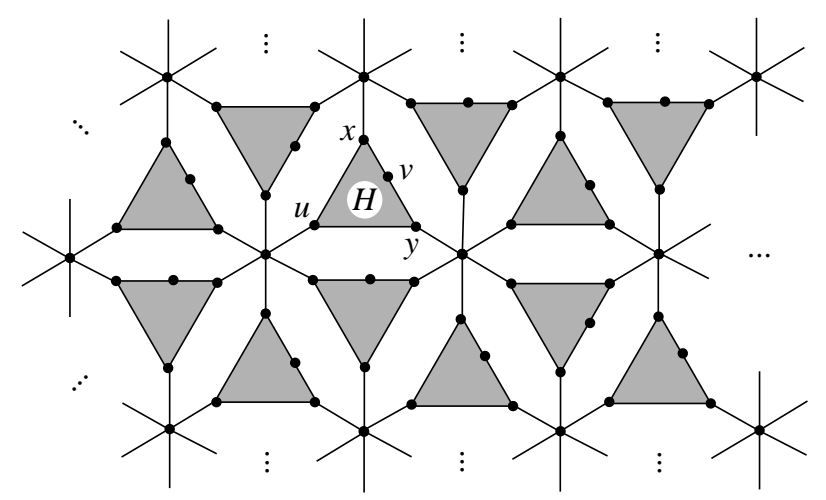

Figure 3: An infinite 3-connected planar graph without a Hamilton cycle

\section{Proof of main result}

Before we start proving Theorem 3, let us reformulate the theorem slightly. The notion of ends are central to the definition of infinite cycles, and we will therefore express the theorem in terms of ends. It is straightforward to see that 
a locally finite graph is $k$-indivisible if and only if it has at most $k-1$ ends. Thus, we obtain the following alternative version of Theorem 3:

Theorem 6. Let $G$ be a locally finite 6-connected planar graph with at most finitely many ends. Then $G$ has a Hamilton cycle.

Our main tool in the proof will be the following result of Thomassen, which itself implies Tutte's theorem:

Theorem 7 (Thomassen [10]). Let $G$ be a finite 2-connected plane graph with a face boundary $C$. Assume that $u \in V(C), e \in E(C)$ and $v \in V(G) \backslash\{u\}$. Then $G$ contains a $C$-Tutte path from $u$ to $v$ and through $e$.

In order to make use of Thomassen's theorem, we need to chop off of the graph all infinite parts, so that a finite part remains, in which we may apply Theorem 7. We then extend this finite Hamilton cycle to a finite part of the chopped off infinite components. For this to work, we need that we can separate off 3-connected infinite components from some arbitrary finite vertex set. This is the task of our next lemma, and, in particular, of its consequence, Lemma 10.

Lemma 8. Let $G$ be a 4-connected locally finite planar graph with minimum degree at least 6 , let $Y \subseteq V(G)$ be a finite vertex set, and let $\omega$ be an end of $G$. Then, there is a finite vertex set $S$ such that for $C_{\omega}:=C(S, \omega)$ holds

(i) $C_{\omega}$ is disjoint from $Y$; and

(ii) if $X \subseteq V\left(C_{\omega}\right)$ with $|X| \leq 2$, then every component of $C_{\omega}-X$ is infinite.

Proof. We view $G$ as a plane graph. Choose a finite vertex set $S$ such that $C_{\omega}:=C(S, \omega)$ is disjoint from $Y$, and such that $\left|E\left(C_{\omega}, G-C_{\omega}\right)\right|$ is minimal with that property.

Suppose there is a set $X \subseteq V\left(C_{\omega}\right)$ with $|X| \leq 2$ such that there is a finite component $K$ of $C_{\omega}-X$. Denote by $K^{\prime}$ the subgraph obtained by adding to $K$ the vertices in $X$ and the edges between $X$ and $K$. In particular, note that any possibly existing edges between vertices in $X$ are not included in $K^{\prime}$. Put $r:=\left|E\left(K, G-C_{\omega}\right)\right|$, and $s:=|E(X, K)|$. We will show that $s<r$. Then $S^{\prime}:=S \cup V(K)$ leads to a smaller cut between $C\left(S^{\prime}, \omega\right)$ and the rest of the graph, a contradiction.

First, assume that $K^{\prime}$ is not a triangulation. Thus, putting $n:=|V(K)|$, Euler's formula implies $\left|E\left(K^{\prime}\right)\right|<3(n+|X|)-6 \leq 3(n+2)-6=3 n$. On the other hand,

$$
2\left|E\left(K^{\prime}\right)\right|=\sum_{v \in V\left(K^{\prime}\right)} d_{K^{\prime}}(v)=|E(X, K)|+\sum_{v \in V(K)} d_{K}(v) \geq s+6 n-r .
$$

For the last inequality recall that the minimum degree of $G$ is at least 6 . Hence, $3 n+(s-r) / 2 \leq\left|E\left(K^{\prime}\right)\right|<3 n$ and thus $s<r$, as desired.

Second, let $K^{\prime}$ be a triangulation. We may assume that some vertices in $S$ lie in the outer face of $K^{\prime}$. Since $G$ is 4-connected and since the face boundary of the outer face of $K^{\prime}$ is a triangle, $T$, say, no vertices of $G$ can be contained in the interior face of $T$. Thus, $T=K^{\prime}$, and consequently the set $X$ consists of exactly one vertex as there are no edges between vertices of $X$ in $K^{\prime}$. This implies that there are exactly two edges between $X$ and $K$, i.e. $s=2$. Since 
the minimum degree is at least 6 in $G$, and as the exactly two vertices in $K$ can have at most one edge between them, it follows that $r \geq 3$. Again, we get $s<r$, as desired.

Lemma 9. Let $G$ be a $k$-connected graph, and let $V(G)=A \cup B$ be a partition such that $G[B]$ is l-connected. Consider $X \subseteq A$ with $|X| \leq k-l$. Then, for every component $K$ of $G[A]-X$ the graph $G[K \cup B]$ is still l-connected.

Proof. Suppose there is a vertex set $Y \subseteq V(K) \cup B$ with $|Y|<l$, so that $G[K \cup B]-Y$ has (at least) two distinct components $C$ and $D$. If both $C$ and $D$ contain vertices of $B$ then $Y \cap B$ separates two vertices of $G[B]$, contradicting that $G[B]$ is $l$-connected. So we may assume that $C \subseteq K$. Then, $X \cup Y$ separates $C$ from $B$ in $G$, but $|X \cup Y|<(k-l)+l=k$, a contradiction.

Lemma 10 will be used in each of the induction steps of the proof of Theorem 6 .

Lemma 10. Let $G$ be a locally finite planar 6-connected graph, let $Y$ be a finite vertex set and $\omega$ an end of $G$. Let $S \subseteq V(G)$ be a finite vertex set such that $C:=C(S, \omega)$ is 2-connected and such that no end other than $\omega$ belongs to $C(S \cup Y, \omega)$. Then there is a finite vertex set $S^{\prime}$ such that $C\left(S^{\prime}, \omega\right) \subseteq C$ is 3 -connected and disjoint from $Y$, and such that $C-C\left(S^{\prime}, \omega\right)$ is 2-connected.

Proof. By adding finitely many vertices from $C$ to the set $(Y \cup N(S)) \cap V(C)$, we can ensure that for the resulting finite set $Y^{\prime} \subseteq V(C)$, the subgraph $G\left[Y^{\prime}\right]$ of $C$ is 2-connected. Applying Lemma 8 to $Y^{\prime}$ and $\omega$ yields a finite vertex set $\tilde{S}$. Clearly, as $D:=C(\tilde{S}, \omega)$ is disjoint from $Y^{\prime}$ it is also disjoint from $Y$. Since $Y^{\prime} \supseteq N(S) \cap V(C)$ it follows that $D \subseteq C$. Moreover, $D$ is 3-connected because of (ii) in Lemma 8 and the fact that there is only end, namely $\omega$, belonging to $D$. Next, observe that since $G\left[Y^{\prime}\right] \subseteq C$ is 2-connected, there is a block $B$ of $C-D$ containing $Y^{\prime}$.

We claim that $N(C-B)=: S^{\prime}$ is finite and that $C\left(S^{\prime}, \omega\right)=C-B$ is 3 connected. As $C-B$ is disjoint from $Y$, because of $Y^{\prime} \subseteq V(B)$, the statement of the lemma follows from this claim.

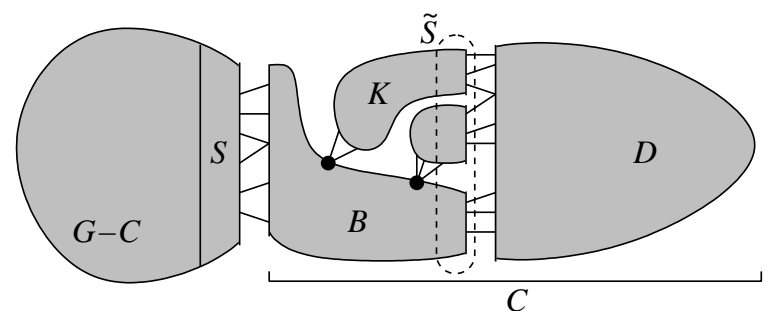

Figure 4: Illustration of the proof of Lemma 10

So, let us prove the claim. The vertex set of $C-B$ consists of vertices in $D$ and of vertices from components of $C-D-B$. Let us consider such a component $K$ of $C-D-B$. Observe that $K$ may have at most one neighbour in $B$ since any path between two vertices of a block lies entirely in the block. Also, $K$ cannot have neighbours outside $C$ as $K$ is disjoint from $Y^{\prime} \supseteq N(S) \cap V(C)$. Thus, all 
neighbours of $K$ in $G$, except possibly one, lie in $D$. Consequently, Lemma 9 (set $X=N(K) \cap V(B)$ ) implies that $G[D \cup K]$ is 3-connected. Moreover, as $G$ is 2-connected, $K$ has a neighbour in $D$, and thus meets the finite set $\tilde{S} \supseteq N(D)$. Therefore, $C-D-B$ has only finitely many components. Now, enlarging $D$, one by one, by the components of $C-D-B$ we see that $C-B$ is 3 -connected.

Finally, it remains to show that $\left|S^{\prime}\right|<\infty$. But this is easy: a vertex in $S^{\prime}$ is either contained in $N(D) \subseteq \tilde{S}$ (which is finite) or it is the single neighbour in $B$ of a component of $C-D-B$, of which, as shown above, there are only finitely many.

We will construct our Hamilton cycle in a piecewise manner. Slightly more precise, we will construct finite nested subgraphs $G_{i}$ of our locally finite graph $G$ in which the application of Theorem 7 will yield subgraphs $H_{i}$ that are finite approximations of the desired infinite Hamilton cycle. The following definition and lemma make sure that these subgraphs $H_{i}$ indeed tend to a cycle, ie. that $\bigcup_{i=1}^{\infty} H_{i}$ is a cycle.

If $G^{\prime}$ is a subgraph of $G$ then denote by $G^{\prime+}$ the subgraph obtained by adding to $G^{\prime}$ the edges in $E\left(V\left(G^{\prime}\right), V\left(G-G^{\prime}\right)\right)$ together with their incident endvertices. We call a sequence $\left(G_{1}, H_{1}\right), \ldots,\left(G_{k}, H_{k}\right)$ of finite induced subgraphs $G_{1} \subseteq$ $\ldots \subseteq G_{k} \subseteq G$ and subgraphs $H_{1} \subseteq \ldots \subseteq H_{k} \subseteq G$ good, if for $i=1, \ldots, k$ holds

(i) $G_{i-1}^{+} \subseteq G_{i}$ for $i \geq 2$;

(ii) $E\left(H_{i} \cap G_{i-1}^{+}\right)=E\left(H_{i-1}\right)$ for $i \geq 2$;

(iii) $V\left(G_{i}\right) \subseteq V\left(H_{i}\right) \subseteq V\left(G_{i}^{+}\right)$;

(iv) if $K$ is an infinite component of $G-G_{i}$ then $\left|E(K, G-K) \cap E\left(H_{i}\right)\right|=2$; and

(v) there is a finite cycle $Z$ such that $Z \cap G_{i}=H_{i} \cap G_{i}$.

Lemma 11. Let $G$ be a locally finite connected graph with at most finitely many ends, and let every finite initial segment of $\left(G_{1}, H_{1}\right),\left(G_{2}, H_{2}\right), \ldots$ be good. Then $H:=\bigcup_{i=1}^{\infty} H_{i}$ is a Hamilton cycle of $G$.

Proof. By (i) it holds that $V(G)=\bigcup_{i=1}^{\infty} V\left(G_{i}\right)$, and hence (iii) implies $V(H)=$ $V(G)$. Therefore, $H$ is a Hamilton cycle if we can prove that it is a cycle at all. For this, we first show that no finite cut $F \subseteq E(G)$ avoiding $E(H)$ separates two vertices of $H$. Suppose, that on the contrary there is such a cut $F$, and that it separates $u, v \in V(H)$, say. Because of (i) we can choose $N$ large enough so that $F \subseteq E\left(G_{N}\right)$ and $u, v \in V\left(G_{N}\right)$. Then a cycle $Z$ as in (v) contains a $u-v$ path in $G$ that is disjoint from $F$ as $E(Z) \cap F=E(Z) \cap F \cap E\left(G_{N}\right)=$ $E\left(H_{N}\right) \cap E\left(G_{N}\right) \cap F=\emptyset$, a contradiction.

Assume that one of the components of $H, C$ say, is a finite cycle. Since the edges between $V(C)$ and $V(G-C)$ then constitute a finite cut that avoids $E(H)$ but separates $C$ from the rest of the graph, we obtain that $H=C$, and we are done.

So, as (v) implies that $H$ is 2-regular, we may assume that $H$ is the disjoint union of double rays. Let the set of these be $\mathcal{D}$. We define a multigraph $H^{\prime}$ with $\Omega(G)$ as vertex set and $\mathcal{D}$ as edge set: each $D \in \mathcal{D}$ will be incident with the ends it contains tails of. In particular, if all tails of $D$ lie in the same end $\omega$ then $D$ becomes a loop at $\omega$. Conditions (ii) and (iv) ensure that $H^{\prime}$ is 2-regular. 
Suppose that $H^{\prime}$ is not connected. Let $X$ be a component of $H^{\prime}$, and set $Y:=H^{\prime}-X$. Choose $N$ large enough so that no component of $G-G_{N}$ has more than one end belonging to it. Because of (iv) this prevents any infinite component of $G-G_{N}$ from meeting both a double ray from $E(X)$ and of $E(Y)$. Contracting each infinite component of $G-G_{N}$ (keeping parallel edges but deleting loops) yields a finite minor $G^{\prime}$ of $G$, in which no edge belonging to a double ray in $E(X)$ is adjacent with an edge lying in a double ray in $E(Y)$. Therefore, there is a cut $F$ of $G^{\prime}$ separating these two sets of edges. This cut then also separates two vertices of $H$ in $G$, contradicting what we have shown earlier. Consequently, $H^{\prime}$ is a cycle, and hence $H$ is an infinite cycle in $G$.

Let us introduce one useful definition before we finally start with the proof. We call a vertex set $U$ in a graph $G$ externally $k$-connected, if $|U| \geq k$ and if for every set $X \subseteq V(G)$ with $|X|<k$ there is a path between any two vertices of $U \backslash X$ in $G-X$.

Proof of Theorem 6. By Theorem $4,|G|$ can be embedded in the sphere. We will identify $|G|$ with that embedding, and thus view $G$ as a plane graph.

Inductively, we will construct connected finite induced subgraphs $G_{1} \subseteq G_{2} \subseteq$ $\ldots \subseteq G$ and subgraphs $H_{1} \subseteq H_{2} \subseteq \ldots \subseteq G$ such that for every $i \geq 1$ it holds that

(a) $\left(G_{1}, H_{1}\right), \ldots,\left(G_{i}, H_{i}\right)$ is good;

(b) if $\mathcal{C}_{i}$ is the set of components of $G-G_{i}$ then each $C \in \mathcal{C}_{i}$ is 3-connected and there is exactly one end belonging to $C$; and

(c) for each $C \in \mathcal{C}_{i}$ it follows that $\left|V(C) \cap V\left(H_{i}\right)\right|=2$.

If this can be achieved, then, by Lemma $11, \bigcup_{i=1}^{\infty} H_{i}$ is a Hamilton cycle.

Before we proceed, let us give a rough outline over the proof. Ideally, we would like to perform infinitely many steps as follows. In the first step, our foremost task is to separate all the ends. For this, we will find a finite subgraph $G_{1}$, so that all components of $G-G_{1}$ are 3-connected and have exactly one end belonging to them. By contracting these components we will obtain a minor of $G$, in which the application of Theorem 7 will deliver us the initial piece $H_{1}$ of the Hamilton cycle. Slightly more precisely, Theorem 7 yields a Tuttepath in the minor - its edges, viewed as edges of $G$, together with the incident vertices in $G$ form $H_{1}$. In the second step, we need to extend $H_{1}$ into each of the components of $G-G_{1}$. We will treat one component after each other, starting with $C$, say. In $C$ we will find a finite subgraph $G_{C}^{\prime}$, so that the unique component $D$ of $C-G_{C}^{\prime}$ is 3-connected. We will use Theorem 7 in the minor obtained from $C$ by contracting $D$. As we will require the resulting Tutte-path $H_{C}^{\prime}$ to start in one of the two vertices in $H_{1} \cap C$ and end in the other (here, note (c)), $H_{C}^{\prime}$ and $H_{1}$ will fit together. Doing this for all components of $G-G_{1}$ we will construct $H_{2} ; G_{2}$ will be defined to be the (induced subgraph of the) union of $G_{1}$ and all the $G_{C}^{\prime}$. Then, we will repeat the second step infinitely often. See Figure 5 for an illustration.

We wrote "ideally" at the beginning of this outline, and that was for a reason: in one aspect the procedure has to be amended in order to work. It is crucial that the $H_{i}$ cover all vertices of the $G_{i}$-otherwise $\bigcup H_{i}$ will never be a 


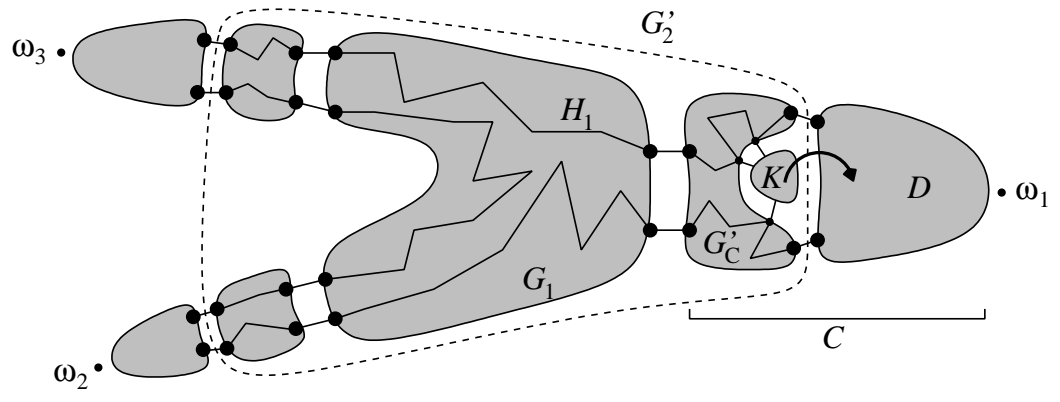

Figure 5: Outline: bridge $K$ is not covered by $\mathrm{H}_{2}$

Hamilton cycle. However, $H_{C}^{\prime}$ will, in general, miss some vertices of $G_{C}^{\prime}$, and thus produce nonchordal $H_{C}^{\prime}$-bridges in $G_{C}^{\prime}$. Fortunately, and here the fact that the components of $G-G_{i}$ are 3-connected will come in handy, we will be able to force such a nonchordal bridge to have all its neighbours in $G_{C}^{\prime} \cup D$. This will allow us to "put the bridge back": it will be merged with $D$ and, thus, covered in later steps.

Let us continue with the proof. As the construction of the base case, i.e. when $i=1$, is quite similar to the general case, i.e. when $i \geq 2$, we will treat both at once. However, for some of the steps we will need to make case distinctions. Put $G_{0}:=\emptyset, H_{0}:=\emptyset$ and $\mathcal{C}_{0}=\{G\}$, and assume $\left(G_{i-1}, H_{i-1}\right)$ to be constructed for some $i \geq 1$. Consider a component $C \in \mathcal{C}_{i-1}$.

First, let $i=1$, and note that as $C=G$ has only finitely many ends, we can choose a finite vertex set $W$ such that no two ends belong to the same infinite component of $G-W$. Denote by $F_{C}$ a face boundary of $|G|$, and pick an edge $x_{C} y_{C} \in E\left(F_{C}\right)$, a vertex $u_{C} \in V\left(F_{C}\right)$ with $u_{C} \notin\left\{x_{C}, y_{C}\right\}$, and a vertex $v_{C} \notin\left\{u_{C}, x_{C}, y_{C}\right\}$ that is adjacent to $u_{C}$. Put $U_{C}:=W \cup\left\{u_{C}, v_{C}, x_{C}, y_{C}\right\}$.

For $i \geq 2$, observe that, by (c), $H_{i-1}$ contains exactly two vertices of $C, u_{C}$ and $v_{C}$ say. By (iii), $u_{C}$ and $v_{C}$ are neighbours of $G_{i-1}$. In the embedding of $\bar{C}$ induced by $|G|$ all the neighbours of the connected graph $G_{i-1}$ that lie in $C$ are contained in the same face boundary $F_{C}$ of $\bar{C}$. Pick an edge $x_{C} y_{C} \in E\left(F_{C}\right)$ such that $x_{C} y_{C} \neq u_{C} v_{C}$, and let $U_{C}$ be the union of $\left\{u_{C}, v_{C}, x_{C}, y_{C}\right\}$ together with all neighbours of $G_{i-1}$ in $C$. Thus, $U_{C} \subseteq V\left(F_{C}\right)$ separates $C$ from the rest of $G$.

For later use, we note that

$$
\begin{aligned}
& \text { if } i=1 \text { then } U_{C} \text { separates the ends of } G \text {, and if } i \geq 2 \text { then } \\
& U_{C} \subseteq V\left(F_{C}\right) \text { separates } C \text { from } G-C
\end{aligned}
$$

and

$$
\left.u_{C} \neq v_{C} \text { and }\left|\left\{u_{C}, v_{C}, x_{C}, y_{C}\right\}\right| \geq 3 \text { (resp. }=4 \text { for } i=1\right) .
$$

Since $C$ is 3 -connected (resp. 4 -connected for $i=1$ ) there are three (resp. four) internally disjoint paths in $C$ between any two vertices in $U_{C}$. Denote by $Y_{C}$ the vertex set of the union of three (resp. four) such paths for each pair of vertices in $U_{C}$. Clearly, $U_{C}$ is externally 3-connected (resp. externally 4-connected for $i=1)$ in $C\left[Y_{C}\right]$. 
Let $\omega_{1}, \ldots, \omega_{n}$ be the ends belonging to $C$. (Note that if $n \geq 2$ then $i=1$.) Setting $S_{1}:=N(C)$ we apply Lemma 10 to $Y_{C}, \omega_{1}, S_{1}$, which yields a finite vertex set $S_{1}^{\prime}$, so that $C\left(S_{1}^{\prime}, \omega_{1}\right)$ is 3 -connected and disjoint from $Y_{C}$, and so that $C-C\left(S_{1}^{\prime}, \omega_{1}\right)$ is 2-connected. Now, proceeding by induction, we set $S_{j}:=S_{j-1} \cup$ $\left(N\left(S_{j-1}^{\prime}\right) \cap V\left(C\left(S_{j-1}^{\prime}, \omega_{j-1}\right)\right)\right)$, and denote by $S_{j}^{\prime}$ the finite vertex set that the application of Lemma 10 to $Y_{C}, \omega_{j}, S_{j}$ yields. Note that $C\left(S_{j}^{\prime}, \omega_{j}\right)$ is 3 -connected and disjoint from $Y_{C}$, and that $C\left(S_{j}, \omega_{j}\right)-C\left(S_{j}^{\prime}, \omega_{j}\right)=C-\bigcup_{l=1}^{j} C\left(S_{l}^{\prime}, \omega_{l}\right)$ is 2-connected. Having done this for $j=1 \ldots n$, we set $G_{C}^{\prime}:=C-\bigcup_{j=1}^{n} C\left(S_{j}^{\prime}, \omega_{j}\right)$ and observe that $G_{C}^{\prime}$ is a finite graph: it is connected but cannot contain any ray, since each ray has a tail in one of the $C\left(S_{j}^{\prime}, \omega_{j}\right)$. Since $C\left[Y_{C}\right] \subseteq G_{C}^{\prime}$ it holds therefore that

$G_{C}^{\prime}$ is a finite 2-connected graph, and $U_{C}$ is externally 3connected (resp. externally 4-connected for $i=1$ ) in $G_{C}^{\prime}$.

Moreover, as $U_{C} \subseteq V\left(G_{C}^{\prime}\right)$ contains all neighbours of $G_{i-1}$ in $C$ it follows that

$$
\begin{aligned}
& G_{i}^{\prime}:=G\left[V\left(G_{i-1} \cup \bigcup_{B \in \mathcal{C}_{i-1}} G_{B}^{\prime}\right)\right] \text { is connected, and every com- } \\
& \text { ponent of } G-G_{i}^{\prime} \text { is } 3 \text {-connected and has exactly one end of } G \\
& \text { belonging to it. }
\end{aligned}
$$

The graph $G_{i}^{\prime}$ will serve as a precursor to $G_{i}$. Note that each component of $G-G_{i}^{\prime}$ is of the form $C\left(S_{j}^{\prime}, \omega_{j}\right)$ for some $j$ and $C \in \mathcal{C}_{i-1}$, and vice versa. To avoid several indices we will from now on denote the set of components of $G-G_{i}^{\prime}$ that lie in a $C \in \mathcal{C}_{i-1}$ by $\mathcal{D}_{C}$. Observe that for $i>1$ it holds that $\left|\mathcal{D}_{C}\right|=1$.

As stated in the outline above, we now want to use Theorem 7 in the minor obtained from $C$ by contracting all elements of $\mathcal{D}_{C}$. Working in this minor, instead of in $G_{C}^{\prime}$, say, has the advantage that we can force the resulting Tuttepath $\tilde{H}_{C}$ to go through each contraction vertex. If $E\left(\tilde{H}_{C}\right)$ is viewed as a subset of $E(G)$ then this means that $E\left(\tilde{H}_{C}\right)$ uses exactly two edges in the cut $E(G-D, D)$ for every $D \in \mathcal{D}_{C}$, which is (iv). However, we need slightly more: we need that these two edges are not incident with the same vertex in $D$ - see Condition (c). To achieve this, we will delete some edges from $E(G-D, D)$ before forming the minor. This is what we will do next.

So, consider a $D \in \mathcal{D}_{C}$. Let $M_{D}$ be obtained from $E(D, G-D)$ by deleting for each vertex in $D$ all but one of the incident edges in $E(D, G-D)$. Thus,

every neighbour of $G_{C}^{\prime}$ in $D$ is incident with exactly one edge of $M_{D}$.

Starting from $G_{C}^{\prime}$ define a 2-connected finite plane graph $\tilde{G}_{C}$ as follows: for every $D \in \mathcal{D}_{C}$ put a vertex $z_{D}$ into the face of $G_{i}^{\prime}$ that contains $D$ and link $z_{D}$ to the vertices in $G_{C}^{\prime}$ that are incident with an edge in $M_{D}$. We will identify these linking edges with the edges in $M_{D}$. Note that the resulting graph, which is a minor of $G$, may have parallel edges. Then, since, for $i=1, F_{C}$ is a face boundary of $|G|$ such that $u_{C}, x_{C}, y_{C} \in V\left(F_{C}\right)$ and since, for $i \geq 2, U_{C} \subseteq V\left(F_{C}\right)$ this also holds for a face boundary $\tilde{F}_{C}$ of $\tilde{G}_{C}$, i.e.

$$
\begin{aligned}
& \text { for } i=1 \text { we have } u_{C}, x_{C}, y_{C} \in V\left(\tilde{F}_{C}\right) \text {, and for } i \geq 2 \text { it holds } \\
& \text { that } U_{C} \subseteq V\left(\tilde{F}_{C}\right) \text {. }
\end{aligned}
$$

We apply Theorem 7 to $\tilde{G}_{C}$, and obtain an $\tilde{F}_{C^{-}}$Tutte path (resp. cycle for $i=1$ ) $\tilde{H}_{C}$ from $u_{C}$ to $v_{C}$ (resp. through $u_{C} v_{C}$ ) and through $x_{C} y_{C}$. (More precisely, 
if $\tilde{G}_{C}$ has parallel edges we first subdivide these before using Theorem 7; the obtained Tutte-path then induces a Tutte-path in $\tilde{G}_{C}$.) From (2) it follows that

$$
\left|V\left(\tilde{H}_{C}\right) \cap U_{C}\right| \geq 3(\text { resp. } \geq 4 \text { for } i=1) .
$$

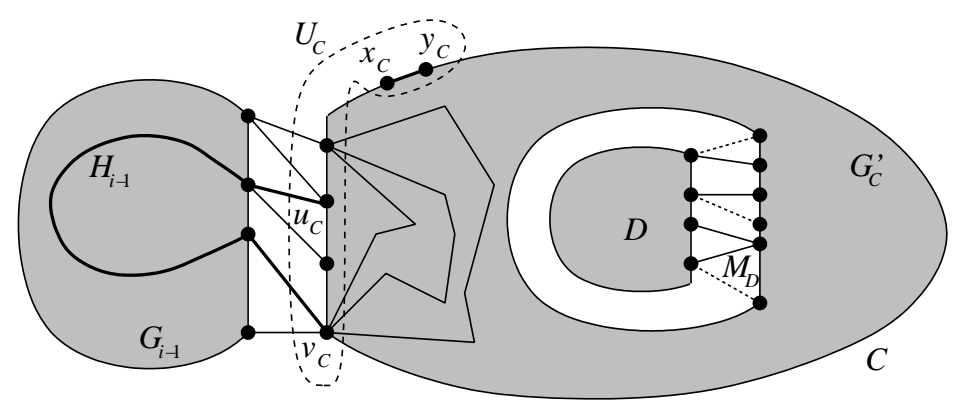

Figure 6: Claims (1)-(5)

Next, we show that

for every nonchordal $\tilde{H}_{C}$-bridge $K$ in $\tilde{G}_{C}, K-\tilde{H}_{C}$ is disjoint from $U_{C}$, and all its neighbours in $G$ lie in $G_{C}^{\prime} \cup D$ for some $D \in \mathcal{D}_{C}$.

Suppose that $K-\tilde{H}_{C}$ meets $U_{C}$. If $i \geq 2$ then $K$ contains an edge of $\tilde{F}_{C}$, as $U_{C} \subseteq$ $V\left(\tilde{F}_{C}\right)$, by (6). Thus, $K$ has at most two (resp. three for $i=1$ ) attachments as $\tilde{H}_{C}$ is an $\tilde{F}_{C}$-Tutte path (resp. cycle). Since $U_{C}$ is externally 3 -connected (resp. externally 4-connected) in $G_{C}^{\prime}$ by (3), this implies $U_{C} \subseteq V(K)$. Thus, by (7), $K-\tilde{H}_{C}$ contains a vertex in $V\left(\tilde{H}_{C}\right) \cap U_{C}$, a contradiction. Therefore, $K-\tilde{H}_{C}$ is disjoint from $U_{C}$.

To prove the second assertion, first assume that $i=1$. Then $C=G$ consists of $G_{C}^{\prime}$ and $\bigcup_{D \in \mathcal{D}_{C}} D$ (plus edges between these graphs). Let there be a neighbour of $K-\tilde{H}_{C}$ in $D \in \mathcal{D}$ (i.e. $\left.z_{D} \in V(K)\right)$. As $U_{C} \subseteq V\left(G_{C}^{\prime}\right)$, all the $D^{\prime} \in \mathcal{D}_{C}$ are disjoint from $U_{C}$. On the other hand, $U_{C}$ separates the ends of $G$, by (1), and thus $D$ from all $D^{\prime} \in \mathcal{D}_{C}, D^{\prime} \neq D$. Consequently, $K-\tilde{H}_{C}$, which is disjoint from $U_{C}$, cannot have neighbours in any $D^{\prime} \in \mathcal{D}_{C}$ other than $D$. If, on the other hand, $i \geq 2$ then $\mathcal{D}_{C}$ consists of only one element, $D$ say. As $G_{C}^{\prime}=C-D$ and as $K-\tilde{H}_{C}$ is disjoint from $U_{C}$, the assertion follows from (1).

It holds that

$$
z_{D} \in V\left(\tilde{H}_{C}\right) \text { for every } D \in \mathcal{D}_{C} .
$$

Indeed, suppose there is a $D \in \mathcal{D}_{C}$ with $z_{D} \notin V\left(\tilde{H}_{C}\right)$. Thus, $z_{D} \in V\left(K-\tilde{H}_{C}\right)$ for some $\tilde{H}_{C}$-bridge $K$. Denote by $f_{D}$ the face of $\overline{G-D}$ that contains $D$. Then there exists a vertex $a$ in the face boundary of $f_{D}$ that does not lie in $V(K)$. Indeed, otherwise the at most three attachments of $K$ separate $D$ from $U_{C} \backslash V(K) \neq \emptyset$ in $G$, contradicting that $G$ is 6-connected. (Note here, that because of (8) and as $z_{D} \in V\left(K-\tilde{H}_{C}\right)$, all vertices of attachment of $K$ are indeed vertices of $G$.)

Next, observe that $a$ is not incident with any edge in $M_{D}$ as $z_{D} \in V\left(K-\tilde{H}_{C}\right)$. Therefore, there is a unique face $f \subseteq f_{D}$ of (the closure of) $(G-D) \cup D \cup M_{D}$ 
whose face boundary $F$ contains $a$. Furthermore, $F$ contains exactly two edges of $M_{D}$, let these be $u c$ and $b w$, where $u, b \in V(D)$; see Figure 7. As $G$ is 6connected there is then a path $P$ between a vertex $v \in V(D)$ and $a$ that avoids $u, b$ and the at most three vertices of attachment of $K$ (which are vertices of $G$, as above). We may assume that $v$ is the only vertex on $P$ in $D$. We note that $v \notin V(F)$ : as the first edge on $P$ lies in $E(D, G-D), v$ is incident with an edge in $M_{D}$, by (5), but the only two vertices in $V(F)$ incident with edges in $M_{D}$ are $u, b$.

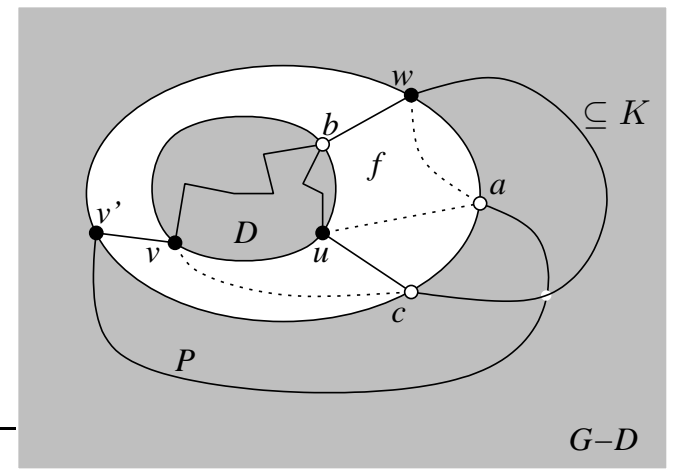

Figure 7: Subdivision of $K_{3,3}$ in (9)

We claim now that by adding some (non-crossing) edges in the plane graph $(G-D) \cup D \cup\left\{u c, b w, v v^{\prime}\right\}$, we obtain a subdivision of $K_{3,3}$ with partition sets $\{a, b, c\}$ and $\{u, v, w\}$, which clearly contradicts planarity. We already have the edges $u c$ and $b w$, and the $v-a$ path $P$. Since all of $a, u, w$ lie in $F$ we can add new edges, drawn in $f$, between $a$ and each of $u, w$. We add another new edge, between $v$ and $c$, which will be drawn in $f_{D} \backslash f$; this is possible since $v \notin V(F)$. As $D$ is 3 -connected, there are two disjoint paths in $D$, one from $b$ to $u$ and the second from $b$ to $v$; these yield two more subdivided edges in the $K_{3,3}$. All that remains now is to find a path from $c$ to $w$. As endvertices of edges in $M_{D}$, both $c$ and $w$ lie in $K$ (because $z_{D} \in V\left(K-\tilde{H}_{C}\right)$ ). Thus, if $K-z_{D}$ is connected (in $G$ ) there will be $c-w$ path in $K-z_{D}$ avoiding all the other subdivided edgesnote that $P$ is disjoint from $K-z_{D}$ as it could only leave $K$ through one of its vertices of attachment.

So, suppose that $K-z_{D}$ has two components. Because of (8), each component must contain a vertex in the boundary of $f_{D}$. As $K$ has at most three vertices of attachment, one of the components, $K^{\prime}$ say, has at most one vertex in the boundary of $f_{D}$. But then this single vertex separates $K^{\prime}$ from the rest of $G$, a contradiction. This establishes (9).

Consider $E\left(\tilde{H}_{C}\right)$ as a subset of $E(G)$, and let $H_{C}$ be the subgraph of $G$ consisting of the edges $E\left(\tilde{H}_{C}\right)$ and the incident vertices. Put $H_{i}:=H_{i-1} \cup$ $\bigcup_{C \in \mathcal{C}_{i-1}} H_{C}$, and observe that the pair $\left(G_{i}^{\prime}, H_{i}\right)$ already satisfies almost all of the desired properties. Clearly, (b) is (4), and (c) holds because of (9) and the definition of $M_{D}$. Moreover, for (a), $\left(G_{1}, H_{1}\right), \ldots,\left(G_{i-1}, H_{i-1}\right),\left(G_{i}^{\prime}, H_{i}\right)$ should be a good sequence, and indeed, it at least satisfies (i), (ii), (iv), (v): (i) holds because of $U_{C} \subseteq V\left(G_{C}^{\prime}\right)$ for every $C \in \mathcal{C}_{i-1}$ (for $i \geq 2$ ), (ii) because no $H_{C}$ uses edges of $G_{i-1}^{+}$, and (iv) because of (9). To see (v), add a path through $D$ 
between the two vertices of $H_{i}$ in $D$ for every $D \in \mathcal{D}_{C}$, and denote the resulting subgraph by $Z$. Clearly, $Z$ is connected, and every $w \in V(Z)$ has degree two: if $w \in V\left(G_{i-1}\right)$ then because of (v) for $i-1$, if $w \in\left\{u_{C}, v_{C}\right\}$ then because $u_{C} \neq v_{C}$, if $w \in V\left(H_{i}-H_{i-1}\right)$ because $H_{i}$ is a disjoint union of paths for every $C$, and finally if $w \in V\left(Z-H_{i}\right)$ because $Z-H_{i}$ is a disjoint union of paths. Thus, $Z$ is a cycle.

However, (iii) may fail. To fix this, consider a nonchordal $H_{i}$-bridge $K$ in $G_{i}^{\prime}$. By (iii) for $i-1$, we deduce that $K-H_{i}$ is disjoint from $G_{i-1}$. Observe that for each $C \in \mathcal{C}_{i-1}, K \cap G_{C}^{\prime}$ is either empty, a chord or a union of $H_{C}$-bridges. Thus, $K-H_{i}$ is disjoint from $U_{C}$, by (8), which implies with Lemma 9 that $G_{i}^{\prime}-\left(K-H_{i}\right)$ still satisfies (i) and (b), and then also (i), (ii), (iv), (v) and (c). Thus, putting $G_{i}:=G_{i}^{\prime}-\left(G_{i}^{\prime}-H_{i}\right)$ we see that for the pair $\left(G_{i}, H_{i}\right)$ conditions (a)-(c) hold.

\section{References}

[1] R. Adomaitis. Infinite Hamiltonian graphs. Preprint 1995.

[2] Q. Cui, J. Wang, and X. Yu. Hamilton circles in infinite planar graphs. Preprint 2007.

[3] R. Diestel. The cycle space of an infinite graph. Comb., Probab. Comput., 14:59-79, 2005.

[4] R. Diestel. Graph Theory (3rd edition). Springer-Verlag, 2005.

[5] R. Diestel and D. Kühn. On infinite cycles I. Combinatorica, 24:69-89, 2004.

[6] R. Diestel and D. Kühn. On infinite cycles II. Combinatorica, 24:91-116, 2004.

[7] A. Georgakopoulos. Infinite Hamilton cycles in squares of locally finite graphs. Preprint 2006.

[8] C.St.J.A. Nash-Williams. Hamiltonian lines in infinite graphs with few vertices of small valency. Aequationes Math., 7:59-81, 1971.

[9] R.B. Richter and C. Thomassen. 3-connected planar spaces uniquely embed in the sphere. Trans. Am. Math. Soc., 354:4585-4595, 2002.

[10] C. Thomassen. A theorem on paths in planar graphs. J. Graph Theory, 7:169-176, 1983.

[11] W.T. Tutte. A theorem on planar graphs. Trans. Am. Math. Soc., 82:99 $116,1956$.

[12] X. Yu. Infinite paths in planar graphs V, 3-indivisible graphs. In preparation.

[13] X. Yu. Infinite paths in planar graphs I, graphs with radial nets. J. Graph Theory, 47:147-162, 2004. 
[14] X. Yu. Infinite paths in planar graphs II, structures and ladder nets. J. Graph Theory, 48:247-266, 2004.

[15] X. Yu. Infinite paths in planar graphs III, 1-way infinite paths. J. Graph Theory, 51:175-198, 2006.

[16] X. Yu. Infinite paths in planar graphs IV, dividing cycles. J. Graph Theory, 53:173-195, 2006.

Henning Bruhn <hbruhn@gmx. net>

Mathematisches Seminar

Universität Hamburg

Bundesstraße 55

20146 Hamburg

Germany

Xingxing $\mathrm{Yu}$ <yu@math.gatech.edu>

School of Mathematics

Georgia Institute of Technology

Atlanta, GA 30332-0160

USA

Version 22 Jun 2008 